\title{
Child Health and Delivery of Care During the COVID-19 Pandemic and Beyond
}

\author{
Joseph L. Mathew ${ }^{1}$ (D) \\ Received: 1 June 2020 / Accepted: 2 June 2020 / Published online: 9 June 2020 \\ (C) Dr. K C Chaudhuri Foundation 2020
}

\section{Introduction}

The COVID-19 pandemic shows no signs of abating. Although pediatric cases are fewer and milder [1, 2], pediatric health care services are severely affected in the country. Professors Lodha and Kabra rightly pointed out that restarting these services is necessary [3]. Several additional issues merit consideration, both with respect to COVID, as well as other child health needs affected by COVID. Unfortunately, there are no ready answers to some of the questions raised here, but collective thinking may find effective solutions.

\section{Issues Related to Pediatric COVID}

\section{COVID Testing}

Most pediatric COVID cases in India were family members of adults who developed COVID. A few children hospitalized for other reasons were incidentally detected when tested prior to surgery/procedures. Very few children with severe acute respiratory illness (SARI) who were tested for COVID turned out to be positive. However, testing continues to focus mainly on SARI, with an additional but limited emphasis on influenza like illness (ILI) [4]. Since most pediatric COVID cases were asymptomatic at presentation and throughout the hospitalization period, testing children with SARI or even ILI may be inefficient and ineffective. The ideal testing strategy for children is yet to be evolved.

Joseph L. Mathew

dr.joseph.1.mathew@gmail.com

1 Department of Pediatrics, Advanced Pediatrics Centre, PGIMER, Chandigarh, India

\section{Significance of a Positive Test}

Most infants/children hospitalized for a positive COVID test, stayed with family members in isolation wards, but remained asymptomatic (despite direct contact with infected, albeit asymptomatic, adults). The reverse situation was also observed, wherein COVID-negative parent(s) caring for COVID-positive infants remained asymptomatic and COVID-negative over 14-28 d. These observations confirm that test positivity is not synonymous with infectivity. This raises supplementary issues including (i) significance of the test result for managing an individual child, (ii) whether testing could be restricted to children of COVID-positive family members with respiratory symptoms, (iii) value of one-time testing, etc.

Some children hospitalized with COVID remained 'positive' for long durations (even $>4 \mathrm{wk}$ ), despite remaining asymptomatic throughout. In some cases, infants and children remained positive even after their adult family members had tested negative on two consecutive days (hence eligible for discharge). The reason(s) for this prolonged period of RTPCR positivity among asymptomatic children is/are unclear.

\section{Significance of a Negative Test}

There are several reasons for the RT-PCR test to be falsely negative in children. These include inappropriate timing of the one-time test, inadequate specimen collection, limitations with test sensitivity, and lack of clear knowledge on how much 'viral load' is associated with a positive test (and whether children have that load). Thus, a negative RT-PCR test may not guarantee the absence of COVID. However, children with SARI who test negative are managed as non-COVID. Some of them develop complications and a few succumb. The challenge is to identify which of these children could have COVID. This necessitates a detailed investigation to identify demographic, clinical and biomarker characteristics that are strongly associated with COVID. If this could be done, such 
children could be managed differently, irrespective of their test result.

\section{Children are Not Small Adults}

Most COVID hospitals care for test-positive infants/children along with adult patients (usually in a separate area). Generally, there is only a skeletal staff familiar with pediatric practice. Such settings tend to extrapolate practices used in adult patients including, (i) frequent investigations for potential biomarkers of disease severity or progression, (ii) exercise associated desaturation testing protocols, (iii) frequent radiologic screening, and (iv) use of treatment options and/or regimens not validated in children.

Among adult patients, potentially useful biomarkers include neutrophil: lymphocyte ratio, serum ferritin, D-dimer etc. Management decisions based on these, even in asymptomatic adults, are associated with better outcomes. However, there are no clear guidelines on their value in children. Personal experience suggests that there was no impact on clinical outcome even when no investigations were done in children. Further, a limited number of children who showed abnormal levels of biomarkers but remained untreated, did not develop any clinical consequences.

Current national [5] and international guidelines [6, 7] have limited emphasis on pediatric issues. Even the guidelines developed by some pediatric scientific societies have a very general focus $[8,9]$. Most of these are consensus or expertbased recommendations. In contrast, one strictly evidencebased guideline examined specific management issues in children [10]. Although there was limited evidence when it was developed in January 2020, the recommendations hold good now also. The recent WHO guidelines included some recommendations for pediatric cases [11].

\section{Challenges for Healthcare Workers Managing Children}

Healthcare workers $(\mathrm{HCW})$ wearing personal protective equipment (PPE) while caring for children with confirmed or suspected COVID face several challenges. Some are generic PPE-related issues (physical discomfort, fatigue, thirst, etc.). Issues unique to pediatric care include difficulty in performing procedures like intravenous cannulation, noninvasive procedures without making children cry, invasive procedures without adequate sedation, etc. These routine procedures become more difficult when personnel have to work alone, without the support of trained pediatric nurses or assistance of family members. Performing aerosol generating procedures is further complicated by additional layers of PPE, compromised visibility, and restricted movements while using protective aerosol boxes.
The inability to use stethoscopes while donned in PPE severely limits detection of wheeze (common in infants and children with SARI), other respiratory sounds, confirmation of endotracheal tube position after intubation, correct deployment of infant feeding tubes, and identification of pneumothorax in ventilated children.

Some institutions do not permit COVID-negative caregivers to stay with infants/children, hence HCW have to take care of all clinical and non-clinical needs.

\section{Investigational Therapeutic and Prophylactic Products}

Currently, multiple clinical trials and research studies are underway to identify options for therapy (medications and procedures) or prophylaxis. Understandably, most of these do not include children. Thus even if these trials establish efficacy and safety of specific products/procedures/vaccine(s), potential benefits will trickle to children after a significant lag period when pediatric studies are conducted.

\section{Innovations and Improvisations in Healthcare Delivery}

The threat of COVID during healthcare delivery stimulated several innovations and improvisations [12]. For example, children presenting with respiratory illness are evaluated by physicians through a glass partition using an intercom system. Physical examination is restricted to inspecting overall appearance, counting respiratory rate, and identifying signs of respiratory distress. This simplistic approach is used to either direct children to COVID screening areas, or send them home with advice.

All hospitals improvised upon the basic design of the 'aerosol box' popularized by a Taiwanese physician [13] making them suitable for various clinical purposes.

Many Indian innovators designed respiratory support devices by mechanizing the compression of ambu bags, although none are suitable for managing COVID patients. None has been tested in children. In contrast, we developed the Artificial Breathing Capability Device (ABCD) which is a low cost, efficacious and safe alternative to manual ventilation in children and adults [14]. To date, it is the only device that has been tested in children [15].

\section{Child Health Issues Beyond COVID}

\section{Impact of Paucity of Services and Potential Solutions}

The prolonged lockdown restricted services to children with chronic conditions. Tele-health (using telephones with video- 
conferencing and/or social media applications) can provide limited support to relatively stable children. However, acute conditions, deteriorating clinical status, emergency situations etc. cannot be managed. There are also challenges in maintaining clinical records, taking responsibility for care without physical examination, obtaining and interpreting investigations, and legal standing in the event of conflicts. Further, tele-health delivery is possible only when professionals are available to provide the service. This may be difficult once routine clinical services resume.

\section{Nutrition Related Issues}

Lack of outdoor activities/physical exercise may be associated with weight gain in children from well-to-do families, whereas lack of food security is expected to result in decline in nutritional status of children in impoverished families.

Although it is unclear how much vitamin D is produced in Indian children through exposure to sunlight, the complete lack of outdoor exposure may result in further decline in levels.

\section{Screen Time}

The combination of staying indoors, educational activities, and lack of outdoor recreation avenues, contribute to increased (unsupervised) screen time. This has well-known direct and indirect effects on physical health, eye health, and mental health, and child safety.

\section{Mental Health}

Investigators are exploring the mental health impact of prolonged quarantine, home-based isolation, and lack of social activities. There are anecdotal reports of increased domestic violence and abuse, as a direct or indirect effect of being home-bound for long periods.

\section{Vaccination}

Routine childhood vaccination has been hit badly during the lockdown. Even institutions that have resumed vaccination, are struggling to cope with social distancing. The impact of delayed/missed vaccines on the burden of vaccinepreventable diseases is hard to guess at this time.

\section{Children in Institutions}

The situation of children living in institutions (such as orphanages, homes, etc.) and those attending day-care facilities, creches etc.; needs careful consideration. On the one hand, social distancing and personal protection norms are hard to apply. On the other hand, there is risk of exposure and rapid spread should an unfortunate outbreak (of any infectious disease) occur.

\section{Resumption of Clinical Services}

There are four broad categories of services based on risk perception for individual patients, other children, and healthcare workers. These include (i) High risk services related to the respiratory system; (ii) Intermediate risk services related to systemic issues where respiratory system could be involved; (iii) Low risk services where respiratory system is not affected; and (iv) No risk issues that can be handled telephonically. A team under the leadership of Prof. S. K. Kabra is currently deliberating on the first category. Similarly, teams need to plan resumption of other services without wasting time or resources.

\section{Child Education and Learning}

Many schools/educational institutions have initiated online teaching programs, but these require considerable skill on the part of teachers. Some schools are trying to inculcate a semblance of normality by incorporating schedules, insisting on uniforms, and ensuring that children activate the video feature during online sessions. However, it is difficult to monitor performance of individual children, make education engaging/interactive, or conduct formal evaluation. There is also the risk that rote-based learning will get further accentuated as there is limited provision for imparting practical skills. Last, but not the least, facets of education 'beyond the textbook' including personality development, character building, teamwork, etc. may get neglected altogether.

Even when schools resume routine schedules (probably after the summer vacations), children will need to maintain social distancing and some form of personal protection (home-made masks, sanitizer solutions, etc.). Manufacture of appropriately sized masks needs to go hand-in-hand with training in proper use, recycling, disposal etc.; as well as etiquette to be followed during coughing, sneezing etc. One wonders whether young children can comply with these restrictions and impositions. Similarly, caregivers in educational institutions (teachers, school staff, transport personnel, etc.) would also need to wear masks. The impact of this on child safety is worrisome.

\section{Impact on Parents/Caregivers}

COVID related restrictions on parents/caregivers include compromised job security, reduced income, food insecurity, stress of being house-bound with limited space/facilities, 
limited social support, and ostracization in the event of becoming a COVID suspect or case. A trickle-down effect on child health is expected.

Many families hiring domestic assistants for childcare (when parents work) are presently reluctant to allow outsiders into their homes. Resumption of services will trigger this dependence on outside help for childcare. Anecdotal reports suggest that some parents are attempting to obtain leave from work (to look after children), using justified as well as other means.

\section{What is the Solution?}

- Pediatricians, policy-makers, and parents need to brainstorm together for potentially viable solutions.

- Meticulous data collection on children screened for COVID (for any reason) is required to identify characteristics predicting a positive test.

- Pediatricians managing COVID positive children should contribute data on individual patients to a central data pool to build a robust national database.

- Child heath stakeholders could identify feasible practices used in adult patients and work towards building the evidence base for other strategies.

- Child health must focus beyond COVID to other healthcare needs, childhood vaccination, newborn care, mental and psychological well-being of children, and child safety.

- Some child health issues may not have manifested yet, but need to be anticipated.

- Multi-disciplinary team-work across various stakeholder domains, with out-of-the-box thinking, is the need of the hour.

\section{Compliance with Ethical Standards}

Conflict of Interest None.

\section{References}

1. Ludvigsson JF. Systematic review of COVID-19 in children shows milder cases and a better prognosis than adults. Acta Paediatr. 2020;109:1088-95.

2. Castagnoli R, Votto M, Licari A, et al. Severe acute respiratory syndrome coronavirus 2 (SARS-CoV-2) infection in children and adolescents: A systematic review. JAMA Pediatr. 2020. https://doi. org/10.1001/jamapediatrics.2020.1467.
3. Lodha R, Kabra SK. COVID-19 pandemic: the way forward. Indian J Pediatr. 2020. https://doi.org/10.1007/s12098-020-033562.

4. Indian Council of Medical Research. Strategy for COVID-19 Testing in India (Version 5, dated 18/05/2020). Available at: https://www.icmr.gov.in/pdf/covid/strategy/Testing_Strategy_v5 18052020.pdf. Accessed 30 May 2020.

5. Government of India Ministry of Health \& Family Welfare Directorate General of Health Services (EMR Division). Revised Guidelines on Clinical Management of COVID - 19. Available at: https://www.mohfw.gov.in/pdf/RevisedNational ClinicalManagementGuidelineforCOVID1931032020.pdf. Accessed 30 May 2020.

6. Bhimraj A, Morgan RL, Shumaker AH, et al. Infectious Diseases Society of America Guidelines on the treatment and management of patients with COVID-19. Available at: https:/www.idsociety.org/ practice-guideline/covid-19-guideline-treatment-andmanagement/. Accessed 30 May 2020.

7. COVID-19 Treatment Guidelines Panel. Coronavirus Disease 2019 (COVID-19) treatment guidelines. National Institutes of Health. Available at: https://www.covid19treatmentguidelines.nih.gov/. Accessed 30 May 2020.

8. Indian Academy of Pediatrics. COVID-19 Information and Resources. Available at: https://iapindia.org/get-latest-guidanceon-COVID-19/. Accessed 31 May 2020.

9. Calvo C, García López-Hortelano M, de Carlos Vicente JC, Vázquez Martínez J; Grupo de trabajo de la Asociación Española de Pediatría para el brote de infección por Coronavirus, colaboradores con el Ministerio de Sanidad; Miembros del Grupo de Expertos de la AEP. [Recommendations on the clinical management of the COVID-19 infection by the new coronavirus SARSCoV2. Spanish Paediatric Association Working Group]. An Pediatr (Barc). 2020;92:241.e1-11.

10. Lui E, Smyth RL, Luo Z, et al. Rapid advice guidelines for management of children with COVID-19. Ann Transl Med 2020 [in press]; https://doi.org/10.21037/atm-20-3754.

11. World Health Organization. Clinical management of COVID-19 interim guidance 27 May 2020. Available at: https://www.who.int/ publications-detail/clinical-management-of-covid-19. Accessed 30 May 2020.

12. Mathew JL, Mathew TL. Invention, innovation and imitation in India- Necessity from COVID-19 pandemic. Ann Nat Acad Med Sci. 2020;56(2) [in press].

13. Lai HY. Aerosol Box. Available at: https://sites.google.com/view/ aerosolbox/design. Accessed 14 May 2020.

14. Mathew JL, Sharma M, Kumar N, Sukesha. Artificial Breathing Capability Device (ABCD): A Novel Life-Saving Device for Resource-Limited Settings. Presentation at $4^{\text {th }}$ Global Forum on Medical Devices, Visakhapatnam, 12-14 December 2018. Available at: https://www.who.int/medical_devices/global_forum/ 73_Artificial_Breathing_Capability_Device_novel_life_saving device_limited_resources.pdf. Accessed 17 May 2020.

15. Artificial Breathing Capability Device. Available at: https://www. youtube.com/watch?v=UuzRc6pr4PM. Accessed 30 May 2020.

Publisher's Note Springer Nature remains neutral with regard to jurisdictional claims in published maps and institutional affiliations. 